\title{
Atherectomy devices: technology update
}

This article was published in the following Dove Press journal:

Medical Devices: Evidence and Research

17 December 2014

Number of times this article has been viewed

Nuri I Akkus'

Abdulrahman Abdulbaki'

Enrique Jimenez ${ }^{2}$

Neeraj Tandon ${ }^{2}$

'Department of Cardiology, Louisiana State University Health Sciences Center Shreveport, Shreveport, LA, USA; 'Department of Cardiology, Overton Brooks VA Medical Center, Shreveport, LA, USA
Correspondence: Nuri I Akkus Louisiana State University Health Sciences Center Shreveport, Division of Cardiovascular Diseases, I50I Kings Hwy, Shreveport, LA 7II30, USA

Tel + I 3186755943

Email iakkus@hotmail.com
Abstract: Atherectomy is a procedure which is performed to remove atherosclerotic plaque from diseased arteries. Atherosclerotic plaques are localized in either coronary or peripheral arterial vasculature and may have different characteristics depending on the texture of the plaque. Atherectomy has been used effectively in treatment of both coronary and peripheral arterial disease. Atherectomy devices are designed differently to either cut, shave, sand, or vaporize these plaques and have different indications. In this article, current atherectomy devices are reviewed.

Keywords: coronary artery disease, peripheral arterial disease

\section{SilverHawk and TurboHawk directional atherectomy systems}

The SilverHawk and TurboHawk plaque excision systems are the two US Food and Drug Administration-approved directional atherectomy devices in use today. Both are approved for use in atherectomy of the peripheral vasculature and are not approved for use in coronary, carotid, iliac, or renal arteries. Directional atherectomy is considered a minimally invasive treatment that removes plaque and restores blood flow in the native artery. Besides removing plaque from the body, the other advantages of directional atherectomy include lack of barotrauma, which decreases the risk of neointimal hyperplasia and lesser risk of dissection. Atherectomy does not preclude the use of surgical bypass at a future time and has the advantage of being able to change the bypass site, if needed.

SilverHawk plaque excision system (ev3 Inc., Plymouth, MN, USA) is a forward cutting directional atherectomy device. This can be used with or without concurrent percutaneous balloon angioplasty and stenting. The device consists of a rotating blade inside a tubular housing with a collection area (nosecone) (Figure 1A and B). This catheter is connected to a battery-driven motor which spins the cutter.

The TurboHawk system is similar except in the number of inner blades. While SilverHawk has one inner blade, TurboHawk has four contoured blades, thus favoring use in highly calcified lesions and more plaque removal per pass. Both devices come in various sizes to enable atherectomy in vessels with diameters of 1.5-7 mm. Atherectomy using the SilverHawk device carries with it the advantage of directional control, making it easier to remove eccentric lesions. As the device is advanced through the lesion, plaque is excised and packed in the nosecone. Different planes of excision are achieved by rotation of the device. Distal embolization remains a major 


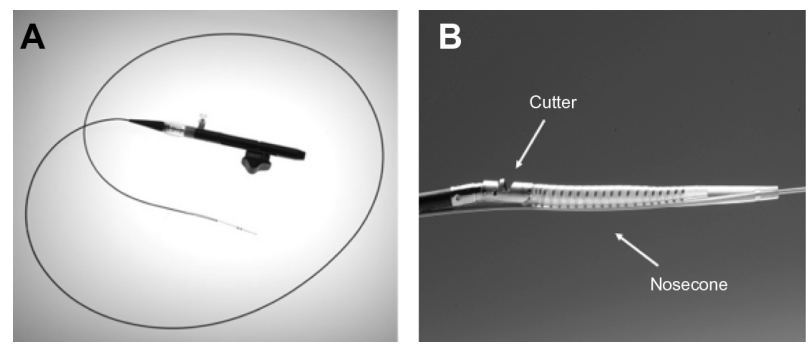

Figure I SilverHawk atherectomy catheter.

Notes: (A) shows the catheter; (B) shows a close-up view of the tip of the catheter, cutter, and nosecone. Reproduced from: Radvany MG, Kiesz RS. Plaque Excision in Management of Lower Extremity Peripheral Arterial Disease with the SilverHawk Atherectomy Catheter. Semin Intervent Radiol. 2008;25(I):I I-19." Images courtesy of Covidien plc, Peripheral Vascular Division, Mansfield, MA, USA.

disadvantage with these systems and hence use of embolic protection devices is recommended in large and heavily calcified vessels.

\section{Clinical data}

The TALON (Treating PeripherAls with SiLverHawk: Outcomes CollectioN) registry enrolled more than 601 patients with over 1,258 infrainguinal arterial lesions that were treated with the SilverHawk device. ${ }^{1}$ It included patients with both claudication and critical limb ischemia (CLI). About half of the patient population was diabetic and a third was Rutherford class 4 or greater. Both above- and below-knee lesions were treated. The device achieved $50 \%$ or less diameter stenosis in $94.7 \%$ of the lesions. The procedural success rate was $97.6 \%$. Stent placement was required in only $6.3 \%$ of the lesions after plaque excision. The 6- and 12-month rates of survival free of target lesion revascularization (TLR) were $90 \%$ and $80 \%$, respectively. Rates of TLR were similar among patients with diabetes (11\%) and without diabetes (9\%). The 12-month outcomes compare favorably to angioplasty and stenting. However, this being an observational registry, it did not have any independent assessment of outcomes.

McKinsey et al, prospectively analyzed 579 lesions treated with SilverHawk in 275 patients with above- and below-knee interventions (claudicants $36.7 \%$, CLI 63.3\%). ${ }^{2}$ Eighteen-month primary and secondary patency was $52.7 \%$ and $75 \%$, respectively. Overall limb salvage was $92.4 \%$ at 18 months with $4.4 \%$ requiring bypass. Kandzari et al prospectively evaluated 69 patients with critical limb ischemia for 6 months after treatment with SilverHawk plaque excision. ${ }^{3}$ A total of 76 limbs were treated with $40 \%$ infrapopliteal lesions. Procedural success was achieved in $99 \%$ of the cases and TLR rate was $4 \%$ at 6 months. Zeller et al reported 1-year and 2-year results after SilverHawk directional atherectomy (DA) of 49 below-the-knee lesions in 36 patients. ${ }^{4}$ Sixty-seven percent of lesions were treated with primary DA, 39\% required additional balloon angioplasty (BA), while $4 \%$ required bail-out stenting. Primary and secondary patency rates were $67 \%$ and $91 \%$, respectively, after 1 year, and $60 \%$ and $80 \%$, respectively, after 2 years. Keeling et al report 1 -year primary and secondary patency of $61.7 \%$ and $76.4 \%$, respectively, from their database of 60 patients in whom 70 plaque excisions were performed. ${ }^{5}$ Restenosis developed in $2.8 \%$ of the patients at 3 months. Sixt et al prospectively treated de novo and restenotic lesions in 161 patients (166 lesions) with plaque excision. ${ }^{6}$ The overall technical success rate was $76 \%$ $(124 / 164)$ and the procedural success rate was $95 \%$. At 12 months, primary patency rate was $61 \%$ and the secondary patency rate was $75 \%$ in the entire cohort. Although SilverHawk is not indicated for treating in-stent restenosis, it has been used for this condition with varying results. Shammas et al studied the effectiveness of plaque excision in management of lower-limb in-stent restenosis with the SilverHawk atherectomy catheter. ${ }^{7}$ They reported from their retrospective analysis of 41 patients that 1-year TLR and target-vessel revascularization occurred in $31.7 \%$ and $34.1 \%$ of cases, respectively. Bailout stenting was used in $24.4 \%$. Distal embolization requiring treatment occurred in $7.3 \%$ and stent thrombosis rate was $4.9 \%$. Another study, DEFINITIVE LE (Determination of Effectiveness of SilverHawk Peripheral Plaque Excision [SilverHawk Device] for the Treatment of Infrainguinal Vessels/Lower Extremities) is a global registry that enrolled patients with both claudication and CLI across 50 sites in the United States and Europe. ${ }^{8}$ This registry is the largest ever conducted, with enrollment of 799 patients worldwide, evaluating a real-world patient population with lesions up to $20 \mathrm{~cm}$ in length and multilevel lesions with the same lesion lengths. Device success was reported at $89 \%$, with a post-atherectomy BA rate of $33 \%$ and bail-out stenting rate of $3 \%$. Rates of distal embolization, dissection, and perforation were $3.8 \%, 2.3 \%$, and $5.3 \%$, respectively. All-complication rate needing treatment was $7.6 \%$. At 12 months, superficial femoral artery patency was $83 \%$ and infrapopliteal artery patency was $78 \%$. Limb salvage rate in CLI patients was $95 \%$. Diabetics were found to perform equally well when compared to nondiabetics. In a study, the analysis of atherectomy samples from peripheral arteries showed $21 \%$ medial and $1 \%$ adventitial component of the arterial wall. ${ }^{9}$ In addition to the reported complications above, SilverHawk atherectomy can cause pseudoaneurysm formation (Figures 2-4), ${ }^{10}$ no flow, and ischemia. ${ }^{1}$ The nextgeneration catheters that contain imaging sensors (optical coherence tomography or intravascular ultrasound) will 


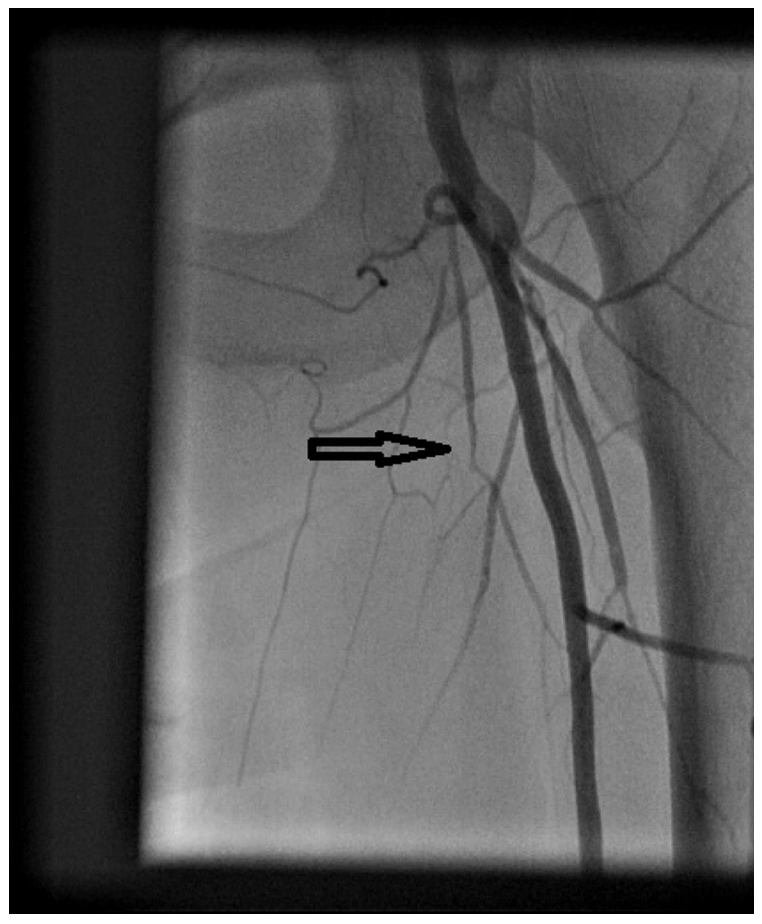

Figure 2 Angiogram showing occluded left superficial femoral artery (arrow).

provide real-time imaging during atherectomy and will help operators to direct the plaque excision. ${ }^{11}$

\section{Rotational atherectomy}

The Rotablator system was developed by David C Auth, $\mathrm{PhD}$ (Boston Scientific Corporation, Redmond, WA, USA)

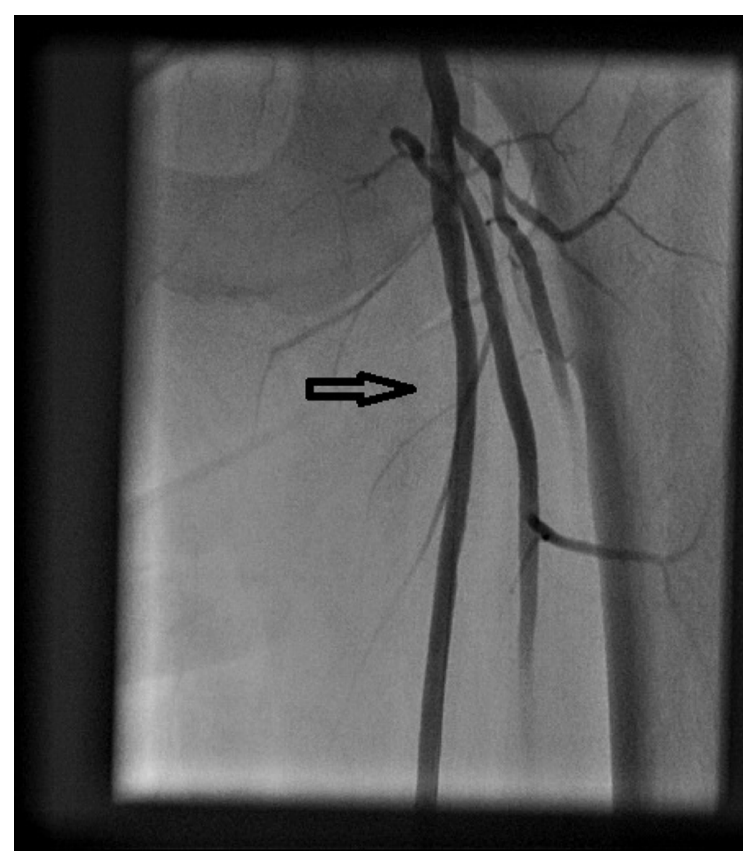

Figure 3 Angiogram showing no significant disease in left superficial femoral artery (arrow) after SilverHawk atherectomy and balloon angioplasty.

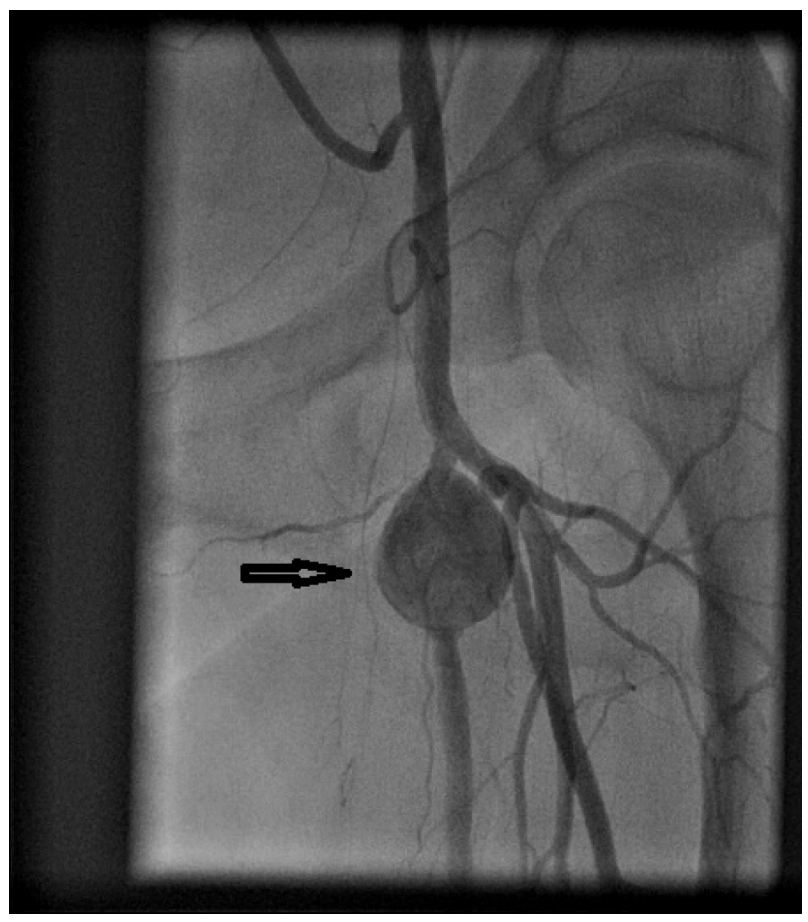

Figure 4 Angiogram showing pseudoaneurysm (arrow) formation in proximal left superficial femoral artery.

and first used in 1988. It is currently available as Rotablator System (Boston Scientific Corporation; Scimed, Plymouth, MN, USA) and consists of an elliptical, nickel-plated, brass burr (Figure 5) which is coated with 2,000-3,000 microscopic diamond crystals on the leading edge. The diamond crystals are $20 \mu \mathrm{m}$ in size, with only $5 \mu \mathrm{m}$ extruding from the nickel coating, and the burr rotates at $140,000-190,000 \mathrm{rpm}^{.12,13}$ The burr is advanced over the 0.009 inch diameter, $325 \mathrm{~cm}$, in length stainless steel RotaWire ${ }^{\mathrm{TM}}$ with a $2.2-2.6 \mathrm{~cm}$ spring tip. The burr should not be advanced close to the spring tip of the wire, and a wire clip torquer should be placed on the wire which will prevent the guide wire from spinning. Available burr sizes are; 1.25, 1.5, 1.75, 2.0, 2.15, 2.25, 2.38, and $2.5 \mathrm{~mm}$. In addition to the burr (which is bonded to the drive shaft), the Rotablator system has a console, a drive shaft, and a turbine (Figure 6). The console is reusable and controls the rotational speed of the drive shaft and burr. The drive shaft is flexible, connected to the turbine, and housed by a 4.3 French Teflon sheath. The Teflon sheath works as a flush delivery conduit and also protects the arterial wall from the spinning drive shaft. ${ }^{12}$ The turbine is driven by compressed air or nitrogen and has the capacity to rotate the shaft and burr at the desired rpm. The turbine is activated by a foot pedal and controlled by the console. ${ }^{12}$ During rotational atherectomy (RA), a cocktail of Rotaglide ${ }^{\circledR}$ lubricant, nitroglycerine, verapamil, and heparin infuses through the Teflon sheath 


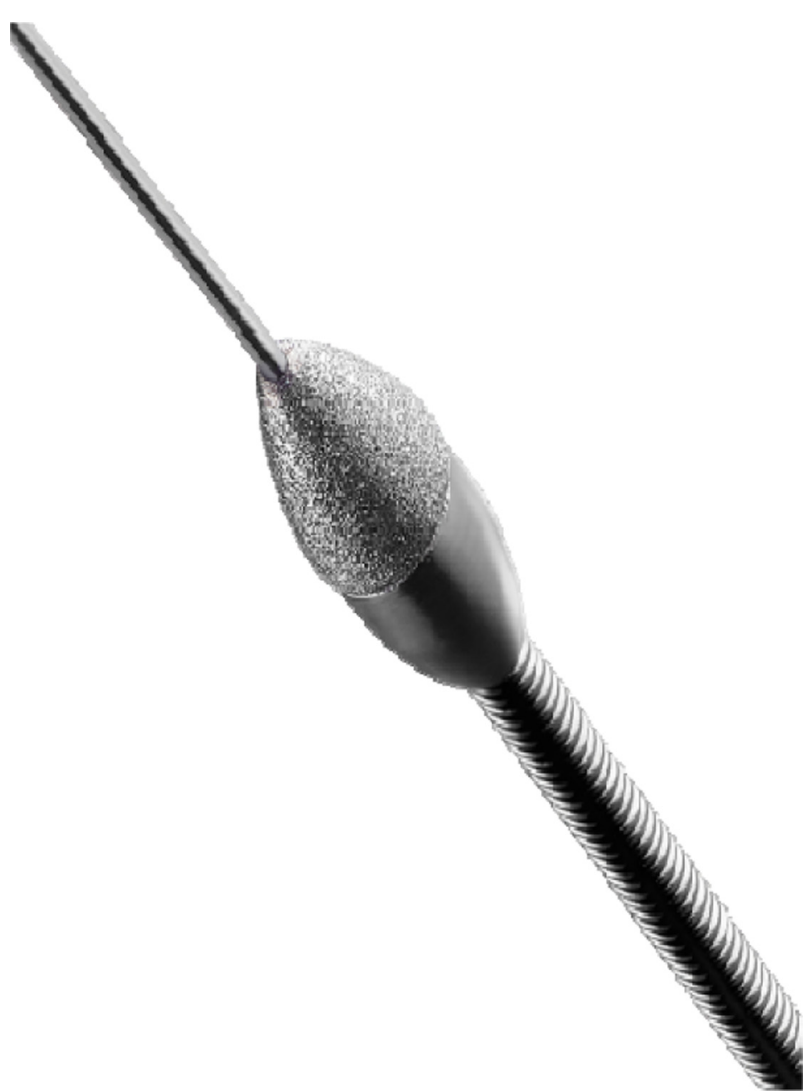

Figure 5 Rotational atherectomy burr.

Notes: Image provided courtesy of Boston Scientific. (c) 2014 Boston Scientific Corporation or its affiliates. All rights reserved. Available from: http://www. bostonscientific.com/en-US/products/plaque-modification/rotablator-rotationalatherectomy-system.html. ${ }^{59}$

which minimizes vasospasm, cools the turbine, lubricates the driveshaft, and flushes the particulate.

High speed rotational ablation differentiates healthy elastic vessel wall from inelastic plaque, and cuts all types of inelastic tissue of plaque morphology (calcium, fibrous

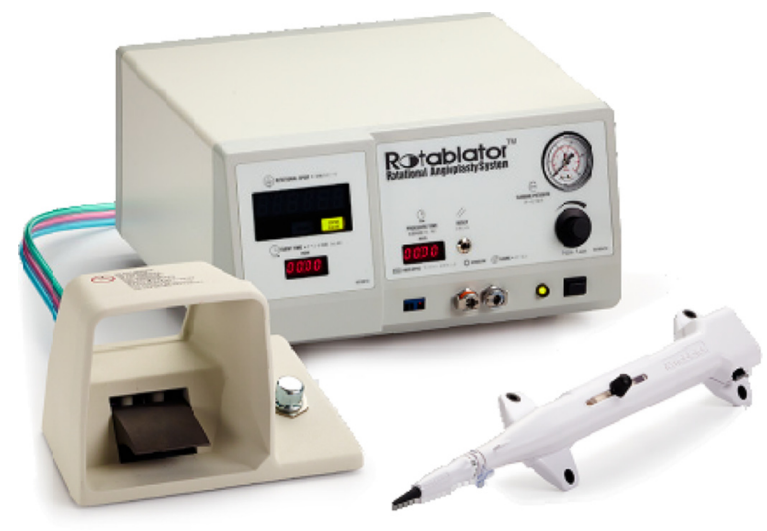

Figure 6 Rotablator system.

Notes: Image provided courtesy of Boston Scientific. (C) 2014 Boston Scientific Corporation or its affiliates. All rights reserved. Available from: http://www. bostonscientific.com/en-US/products/plaque-modification/rotablator-rotationalatherectomy-system.html. ${ }^{60}$ tissue, fatty deposits, restenotic tissue). Healthy tissue has elastic properties and can deflect from the cutting edges of the diamond microchips. In addition to differential cutting, with the high speed rotation, longitudinal friction vector between the guide wire and the device changes to circumferential direction (orthogonal displacement of friction), and both of these properties facilitate the burr advancement through tight, tortuous vessels and lesions. Ninety-eight percent of the plaque debris generated by RA is smaller than $10 \mu \mathrm{m}$, which traverses the coronary microvasculature and is cleared by the reticuloendothelial system. ${ }^{13,14}$

A single burr approach is preferred with a burr-toartery ratio of $0.5: 0.6$, which is more preferable for plaque modification rather than maximum debulking. The turbine is activated by stepping on the pedal until a burr speed of $140,000-150,000 \mathrm{rpm}$ is achieved. The burr is then advanced very gently with a pecking motion into the lesion for intervals of not longer than 15-20 seconds. Friction causing decelerations of the burr of more than 5,000 rpm are to be avoided (by backing out of the lesion) to prevent excessive heat production, vessel injury, platelet activation, and large particles production. ${ }^{14}$

\section{Clinical data}

In the single center Excimer Laser, Rotational Atherectomy, and Balloon Angioplasty Comparison (ERBAC) study, 685 patients were randomized to various atherectomy methods. RA had the greatest initial success - 89\% (RA) versus $77 \%$ (excimer laser) versus $80 \%$ (BA) - but no difference was observed in major in-hospital complications and at the 6-month follow-up. Revascularization of the original target lesion was performed more frequently in the RA group $(42.4 \%)$ and the excimer laser group (46.0\%) than in the angioplasty group $(31.9 \%, P=0.013) .{ }^{15}$ In the multicenter, prospective, Comparison of Balloon Angioplasty versus Rotational Atherectomy in Complex Coronary Lesions (COBRA) trial, 502 patients were enrolled and similar results were found. ${ }^{16}$

Currently, due to lack of benefit in preventing restenosis in native and restenotic lesions, RA is used to prepare the calcified lesions for stenting (drug eluting stents) when the stent is not deliverable/expandable or the lesion is not dilatable with conventional BA. ${ }^{13}$ The absolute contraindications to RA are: saphenous vein graft lesions; presence of thrombus; dissection; and inability to cross the lesion with the guide wire. Relative contraindications include: lesion length of more than $25 \mathrm{~mm}$; lesion angulation of more than $45^{\circ}$; severe left ventricular dysfunction; severe triple vessel disease or 
unprotected left main disease; and no candidacy to coronary artery bypass surgery, either because of patient ineligibility or lack of onsite surgical backup. ${ }^{13,17}$ Some of the reported complications of RA are: Q wave myocardial infarction (MI) $(0.8 \%)$, urgent coronary artery bypass surgery $(2.0 \%)$, non-Q MI (8.9\%), acute closures (1.1\%), slow flow (2\%), perforation $(1.0 \%)$, side-branch closure (5\%), dissection (4\%), and spasm (5\%). ${ }^{18,19}$ Peripheral Rotablator atherectomy has also recently started being used for calcified below-knee arteries. There is no published outcome data available yet about its use in peripheral arterial disease.

\section{Pathway Jetstream PV Atherectomy System}

Pathway Jetstream PV Atherectomy System (Pathway Medical Technologies, Inc., Redmond, WA, USA) consists of a singleuse catheter with control pod and a reusable, compact console power source which can be placed on a standard intravenous stand. The system is indicated for both thrombectomy and RA by the same catheter. The catheter is advanced over the $0.014^{\prime \prime}$ wire with a maximum rate of $1 \mathrm{~mm} /$ second to avoid significant drops in rotational speeds; it has a front-cutting tip that makes it go through tight lesions without predilation. The electric motor spins catheters at 60-70 krpm, and for every 40 seconds of treatment, a 10-second pause in device activation is recommended. ${ }^{20}$ During treatment, saline solution is delivered to the proximal end of the catheter using two lines: one line to flush the motor assembly to maintain an airtight seal, maximizing embolic protection; the other line to infuse saline solution in the treatment area through ports located on the distal body of the catheter to facilitate the catheter's debulking and aspiration capabilities. ${ }^{20}$ Its differentially cutting catheter tip preferentially removes both hard and soft diseased tissue from peripheral arteries with minimal damage to the vessel wall. The rotational design potentially leads to concentric lumens, which can facilitate laminar flow. JETSTREAM ${ }^{\circledR}$ expandable catheters $2.1 \mathrm{~mm} / 3.0 \mathrm{~mm}$ and $2.4 \mathrm{~mm} / 3.4 \mathrm{~mm}$ have a catheter tip that remains at a defined nominal diameter ( $2.1 \mathrm{~mm}$ or $-2.4 \mathrm{~mm}$ ) when spinning clockwise (blades within fenestrated metal housing at the tip of the catheter used), but expands to a defined maximum diameter $(3.0 \mathrm{~mm}$ or $3.4 \mathrm{~mm}$, respectively) when rotating counterclockwise (blades that are hinged and mounted just proximal to the distal housing used) (Figure 7); these sizes are indicated for above-knee arteries. ${ }^{20}$ For the below-knee use, there are fixed cutters (single cutter) with sizes of $1.6 \mathrm{~mm}$ and $1.85 \mathrm{~mm}$. Pathway Jetstream is the only atherectomy device to offer continuous active aspiration, and actively removes atherosclerotic debris and thrombus

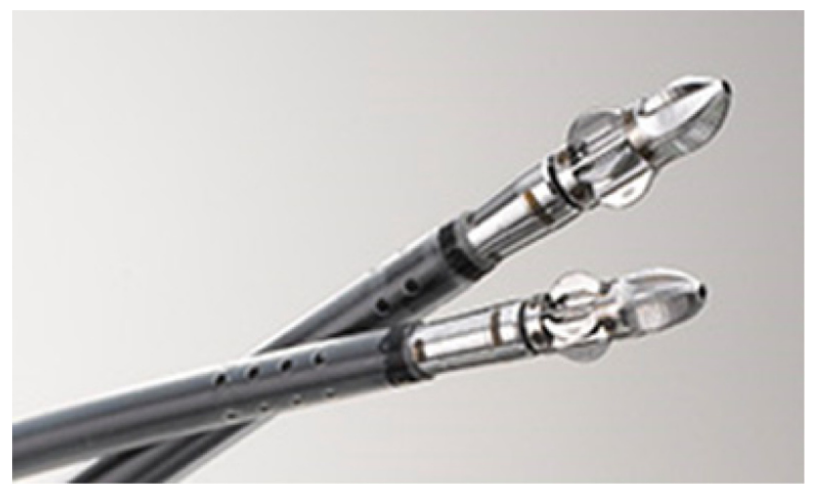

Figure 7 JETSTREAM ${ }^{\circledR}$ expandable catheters.

Notes: Image provided courtesy of Boston Scientific. (c) 2014 Boston Scientific Corporation or its affiliates. All rights reserved. Available from: http://www.medrad. com/en-us/info/products/Pages/jetstream-xc-systems.aspx. ${ }^{61}$

from the treatment site and delivers it to a collection bag located on the console.

\section{Clinical data}

Zeller et $\mathrm{al}^{21}$ used Pathway Jetstream PV Atherectomy System in treatment of 172 patients - 210 lesions (femoropopliteal and infrapopliteal vessels) - with 99\% device success, and 6-month and 12-month target-lesion revascularization rates of $15 \%$ and $26 \%$, respectively. The 1 -year restenosis rate was $38.2 \%$ based on duplex imaging. ${ }^{21}$ In a six-patient intravascular ultrasound study, after Pathway PV use, the lumen area was greater than burr-sized lumen expectancy at cross-sections in the treated segments, which suggested a complementary role of aspiration in luminal gain achieved with this device. ${ }^{22}$ Another intravascular ultrasound study showed substantial plaque volume reduction by removing fibrotic and fibro-fatty plaque by Jetstream atherectomy, which resulted in substantial luminal volume expansion without concomitant vessel expansion with no appreciable effect on necrotic core and dense calcium. ${ }^{23}$

Abrupt vessel occlusion, dissection, distal emboli, hematoma at access site, infection, perforation, pseudoaneurysm, renal failure, restenosis, and thrombus formation are some of the reported complications of Jetstream atherectomy. ${ }^{24}$ In a review of 2,137 lesions treated in 1,029 patients, Jetstream (Pathway Medical Technologies Inc. Kirkland, WA, USA.) and DiamondBack $360^{\circ}$ (Cardiovascular Systems Inc., Saint Paul, MN, USA) devices had a combined embolization rate of $22 \%$ (eight of 36), four of 18 (22\%) in each group, which was significantly higher than with BA alone (five of 570, 0.9\%), BA and stent (five of 740, 0.7\%), SilverHawk atherectomy (14 of 736, $1.9 \%$ ), and laser atherectomy (two of $55,3.6 \% ; P<0.001$ ). ${ }^{25}$ The use of embolization protection may be beneficial when using this device. 


\section{Excimer laser atherectomy}

Laser is an acronym for light amplification by stimulated emission of radiation. Although the history of laser begins in 1951, the first medical application is reported by Goldman in 1962 and used in 1963 for the experimental ablation of atherosclerotic plaques. ${ }^{26}$ The first clinical applications were performed by Choy and Ginsburg in 1983. ${ }^{26}$

Laser atherectomy uses the high energy, monochromatic light beam to alter or dissolve (vaporize) the plaque without damaging the surrounding tissue. Fiber-optic catheters are used to deliver this light beam. For endovascular applications, xenon chloride excimer laser is used and its fiber-optic catheter has multiple small fibers, rather than just a few large fibers, in order to be flexible enough to navigate in the arterial tree. ${ }^{27}$

Laser sources can vary depending on the wavelength of their emitted light, how the light is transmitted (pulsed or continuous), and the effective power of the light beam. In addition to the source, effectiveness of a given laser depends on how the light interacts with the tissue. The absorption depth is determined by the wavelength and the tissue. In the near-infrared regions (2,000 to $3,000 \mathrm{~nm}$ wavelength) the light penetration depth is roughly $1-0.1 \mathrm{~mm}$, whereas in the ultraviolet (UV) B region with a shorter wavelength $(300 \mathrm{~nm})$, the absorption depth is less. For example, at $308 \mathrm{~nm}$, where the XeCL excimer laser emits, the typical absorption depth is about $0.05 \mathrm{~mm}(50 \mu \mathrm{m}) .{ }^{28} \mathrm{UV}$ B light has another advantage in that it uses direct photochemical lytic affect to break molecular bonds rather than thermal affect. ${ }^{29} \mathrm{UV}$ B photons absorbed by the proteins and lipids in cells actually break chemical bonds which facilitates lyses of cellular structures. ${ }^{30}$

In the past, neodymium-yttrium aluminum garnet laser (1,060 $\mathrm{nm}$ wavelength) and the argon laser (500 nm wavelength) with deeper penetration levels were used with a constant/continuous power output and resulted in excess thermal damage, leading to thrombosis, vasospasm, and high restenosis rates. ${ }^{28,31-33}$ On the other hand, with the excimer laser (with less of a penetration depth), the high energy is delivered with short interaction time (pulsed). In this way, chemical bonds are broken only in the tissue that the laser is touching without damaging surrounding material or increasing the heat. ${ }^{28}$

While using laser, pulse width is also important in order to keep the energy delivered to the tip of the catheter faster than the time it will take for the heat to diffuse away from its tip, so that the effect will be only localized to the tissue on contact without a consequent rise in temperature to surrounding tissue. ${ }^{28}$ The excimer laser catheter removes tissue with a thickness of $10 \mu \mathrm{m}$ with each pulse of energy. ${ }^{29}$

There is a unique threshold of energy density for each laser type. If more energy is given, more reaction will be created in the tissue (in expense of more heat generation) which can be used as an advantage in particularly fibrotic or calcified lesions. When laser is used, two factors are controlled by the operators: number of pulses per second (frequency) given and energy amount (fluence). ${ }^{28}$ Pulsed-wave xenon chloride laser (CVX-300; Spectranetics, Colorado Springs, CO, USA) is commonly used in clinical practice; it operates within a wavelength of $308 \mathrm{~nm}$, with relatively long pulses (pulse duration of 135 nanoseconds), and produces an output of $165 \mathrm{~mJ}$ per pulse. ${ }^{27,34}$ The long pulse length is required for successful delivery of the UV light by silica fibers at the fluences necessary for therapy - typically between 30 and $80 \mathrm{~mJ} / \mathrm{mm}^{2}$. After this pulse of 135 nanoseconds, laser energy is not emitted. Typically, pulse repetition rates of 25-40 pulses/second are used. By doing this, the total power emitted from the catheter tip is less than $3 \mathrm{~W}$ for the largest catheters and this minimizes thermal effects during the tissue-ablation process. ${ }^{27}$ The size of the laser catheters used are chosen based on reference coronary/peripheral vessel diameter, and available sizes are $0.9 \mathrm{~mm}, 1.4 \mathrm{~mm}, 1.7 \mathrm{~mm}$, $2.0 \mathrm{~mm}, 2.3 \mathrm{~mm}$, and $2.5 \mathrm{~mm}$.

While using excimer laser, advancement of the catheter should be slow $(0.5 \mathrm{~mm} / \mathrm{second}$ and no faster than $1 \mathrm{~mm} /$ second) for effective plaque removal due to shallow penetration energy depth $(35-50 \mu \mathrm{m}){ }^{31}$ Slower advancement will also create a larger and smoother channel. Since iodinated dye absorbs the excimer laser energy nearly completely and will cause cavitation bubbles, vapor bubbles, and percussive waves which will lead to dissections/perforations, ${ }^{35,36}$ laser catheter should never be activated in contrast media. In addition to angiographic contrast media, blood (hemoglobin) strongly absorbs excimer laser light at $308 \mathrm{~nm} \cdot{ }^{37}$ In order to remove blood and contrast, saline flushes need to be given before and during advancement of the laser catheter so that both will be removed from the artery. The application of saline infusion has resulted in improvements in both angiographic and clinical outcomes. ${ }^{31,37}$ Excimer laser can also vaporize thrombi, suppressing platelet aggregation while ablating the underlying plaque. ${ }^{38}$ There are newer laser catheter designs such as eccentric laser catheters (fiber-optic bundle disposed opposite the guide-wire lumen), which are optimally spaced concentric catheters (fiber bundle placed concentrically around the guide-wire lumen) designed to achieve maximal debulking in complex lesions. ${ }^{27}$ 


\section{Clinical data}

Laser can be used in both coronary and peripheral applications. In coronaries, excimer laser can be used to remove thrombi; to vaporize procoagulant reactants in addition to debulking the underlying plaque; and to facilitate stent delivery. ${ }^{39} \mathrm{In}$ the CARMEL multicenter study, excimer laser angioplasty was successfully used in more than $90 \%$ of the enrolled 151 acute myocardial infarction (AMI) patients with a large thrombus burden with a relatively low rate $(8.6 \%)$ of major cardiac adverse events (MACE) ${ }^{40}$ It has been also used for in-stent restenosis: in the Laser Angioplasty for Restenotic Stents multicenter registry (LARS), laser angioplasty reduced 30-day repeat-target-site coronary intervention, but it did not decrease in 1 year. ${ }^{41}$ Balloon-resistant lesions, chronic total occlusions, calcified lesions, and underexpanded stents in calcified lesions are some other scenarios in which excimer laser coronary atherectomy can be successfully used. ${ }^{42,43} \mathrm{In}$ the CORAL study, ${ }^{44}$ excimer laser atherectomy was used in diseased vein grafts with comparable 30-day MACE (18.4\%) to that of the control population (19.4\%) from the SAFER trial. ${ }^{45}$ It is important to notice that use of a distal embolic protection device in the SAFER trial, where the filter wire was used, showed a reduction of MACE ( $42 \%$ relative reduction) compared to the control group, ${ }^{45}$ which further supports the use of the protection device.

Excimer laser can also be used to assist endovascular treatment of peripheral arterial disease. In the Laser Angioplasty for Critical Limb Ischemia (LACI) trial, 155 critically ischemic limbs with above- or below-knee disease that were poor candidates for surgical revascularization were treated with excimer laser-assisted intervention. Despite the fact that mean treatment length was $>16 \mathrm{~cm}$ and most of the patients had multiple stenosis/occlusions, a limb-salvage rate of $93 \%$ was achieved at 6 months. ${ }^{46}$

The excimer laser has been also used to facilitate crossing of the wire through chronic total occlusions by using the "step-by-step" technique in which the guide wire is advanced just proximal to the lesion, and the excimer laser catheter is advanced to the cap and used briefly to penetrate the fibrous cap ( $<5 \mathrm{~mm}$ distance); then the guide wire is advanced again in the lesion until the wire cannot go further; then the laser is used again to penetrate; then these steps are repeated until the lesion is crossed. ${ }^{46}$ In the study by Scheinert et al, ${ }^{47}$ there were 411 superficial femoral artery (SFA) long-segment occlusions, the average lesion length was $19.4 \mathrm{~mm}$ and, in addition to the technique described above, the guide wire crossed the lesion traditionally and the laser catheter was advanced over the wire. The technical success was $90.5 \%$, with complication rates of $1 \%$ acute reocclusion, $2.2 \%$ perforation, $3.9 \%$ distal embolization, and with 1-year assisted primary and secondary patency rates of $65.1 \%$ and $75.9 \%$, respectively. ${ }^{46,47}$ The distal embolization risk with laser use in the lower extremity is comparable to the risk after angioplasty and stenting. ${ }^{48}$

There are newer laser catheter designs for ablating a larger lumen, such as the TURBO-Booster catheter (Spectranetics) which uses a custom guide catheter that allows the laser to move in different directions and ablate more tissue. The device was studied in the multicenter clinical trial ClirPath Excimer Laser to Enlarge Lumen Openings (CELLO) with high procedural success rate, greater stenosis reduction, and less need for 1-year target-lesion revascularization (was not required in $76.9 \%$ of CELLO participants). ${ }^{49}$

\section{Orbital atherectomy}

Orbital atherectomy $(\mathrm{OA})$ is another atherectomy device being used for plaque modification, to reduce the total atheroma burden, to change the arterial compliance, and to decrease the vessel-wall trauma. ${ }^{50}$ The Diamondback $360^{\circ}$ OAS is very similar to RA devices, as it uses a crown that is equivalent to the RA burr. The crown is eccentrically mounted, diamond coated, and rotates at speeds varying from 60,000 to $200,000 \mathrm{rpm}$. The crown may be advanced forward and backward using a handle once it is intra-arterial. Diamondback $360^{\circ}$ OAS uses its unique orbiting action to remove plaque, and it has the ability to increase lumen diameter by increasing the orbital speed. ${ }^{51}$ The OA crown is eccentric in shape, in contrast to an RA burr. Therefore, the crown orbits on the wire rather than spinning concentrically. As the crown rotates and orbit increases, the diamond-coated crown presses against the lesion or plaque creating a sanding action while removing plaque with each orbit. Theoretically, the less diseased, more elastic arterial wall flexes away from the crown and minimizes the risk of vessel trauma. ${ }^{51}$ Perhaps the most-unique feature of OA is the ability to create variable lumen size with the same catheter by only changing the speed of rotation. Since centrifugal force is a function of both the mass of the device and the speed of rotation, faster speeds result in increased centrifugal force, yielding a larger orbit. As a result, a larger lumen can be created with a given crown by rotating it at higher speeds, which may minimize procedure time and the need for catheter upsizing.

Some other advantages of the orbital motion of the crown is its being in contact with only one part of the vessel wall at any given moment, and not obstructing flow in a diseased vessel ( $100 \%$ occluded vessel which does not have flow anyway), which will minimize heat generation and also lead to the 
continuous clearance of the sanded microscopic particulate matter rather than having it build up into a large load of matter (and micro embolization), which can be seen when the central RA catheter is disengaged from the plaque.

OA system has classic, solid, and micro solid crowns (Figure 8) suitable for different vessels and lesions. The solid crown has more weight and can achieve larger lumens for larger-sized vessels. Crowns come in sizes of 1.25, 1.5, 2.0, and $2.25 \mathrm{~mm}$. The crowns need to be advanced over the wire. As a guide wire, ViperWire ${ }^{\mathrm{TM}}$ (Cardiovascular Systems, Inc.) is used. ViperWire ${ }^{\mathrm{TM}}$ is spring-tip $0.014^{\prime \prime}$ wire, is constructed of stainless steel with a smooth finish, and is silicone coated. ViperSlide ${ }^{\circledR}$ Lubricant and saline infusion are also attached to the system and continuously infused during atherectomy runs to increase lubricity and to decrease friction.

\section{Clinical data}

The Diamondback $360^{\circ}$ OAS peripheral and coronary atherectomy systems are used in diseased peripheral, coronary arteries and in diseased artificial arteriovenous dialysis fistulae. The newer 4 French lower profile and short $60 \mathrm{~cm}$ shaft options may also be preferable in selective tibiopedal access cases. OA has been studied in patients with peripheral and coronary artery disease. CONFIRM registry series (I, II, and III) were planned to evaluate the use of OA in peripheral lesions of the lower extremities. From October 2009 to June 2011 , 3,135 patients undergoing OA by more than 350 physicians at over 200 US institutions were enrolled on an "all-comers" basis. In these registries, treatment with OA reduced preprocedural stenosis from $88 \% \pm 12 \% \mathrm{~s}$ to an average of $10 \%$ with adjunctive treatments, typically lowpressure BA. ${ }^{52}$ Plaque removal was most effective for severely calcified lesions and least effective for soft plaque. Further analysis showed that shorter spin times and smaller crown sizes significantly reduced procedural complications, which

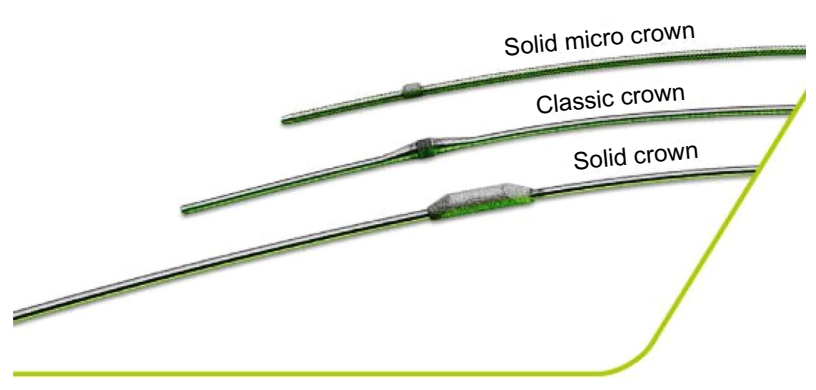

Figure 8 Classic, solid, and micro solid crowns of the Orbital Atherectomy System. Notes: Copyright (c) 2014 Cardiovascular Systems Inc. All Rights Reserved. Reproduced from: Optimal Versatility: A Crowning Achievement [webpage on the Internet]. Saint Paul: Cardiovascular Systems Inc.; 20I4. Available from: http://www. csi360.com/peripheral-stealth-360-peripheral-orbital-atherectomy-system-crowns. ${ }^{62}$ included slow flow (4.4\%), embolism (2.2\%), and spasm $(6.3 \%)$, suggesting more emphasis on plaque modification with OA rather than maximizing luminal gain. ${ }^{52}$

The COMPLIANCE $360^{\circ}$ trial $^{53}$ compared BA combined with $\mathrm{OA}$ to $\mathrm{BA}$ alone in 55 patients with calcified femoral popliteal disease. OA plus BA yielded better luminal gain by improving lesion compliance and decreased adjunctive stenting in the treatment of calcified femoral-popliteal disease, and the OA group required lower balloon inflation pressures compared to the BA arm (4 versus $9.1 \mathrm{~atm}) .^{53}$

In the OASIS trial, OA was used in infrapopliteal disease in 201 stenoses and was shown to provide predictable, safe lumen enlargement with symptomatic improvement and infrequent need for further revascularization or amputation. ${ }^{54}$ The CALCIUM 360 trial also enrolled calcified infrapopliteal arteries in 50 patients with critical limb ischemia and compared OA with BA to BA alone. ${ }^{55}$ The OA appeared to increase the chance of reaching a desirable angioplasty result, with less acute need for bailout stenting and a higher procedure success. ${ }^{55}$

On the coronary system, the Orbital atherectomy system in treating calcified coronary lesions (ORBIT) I trial evaluated the safety and performance of an OA system in treating de novo calcified coronary lesions. Among 50 enrolled patients, device success was $98 \%$, and procedural success was $94 \%$. The cumulative major adverse in-hospital cardiac event rate was 4\% (two non-Q-wave myocardial infarctions). Angiographic complications were observed in seven patients (six dissections and one perforation). ${ }^{56}$

ORBIT II $^{57}$ was a prospective, multicenter, non-blinded clinical trial that enrolled 443 consecutive patients with severely calcified coronary lesions at 49 US sites and used OA system diamond-coated crown to modify calcified lesions prior to stent placement. Preprocedure mean minimal lumen diameter of $0.5 \mathrm{~mm}$ increased to $2.9 \mathrm{~mm}$ after the procedure, with successful stent delivery in $97.7 \%$ of cases and low rates of in-hospital Q-wave myocardial infarction $(0.7 \%)$, cardiac death $(0.2 \%)$, and target-vessel revascularization $(0.7 \%)$.

The use of orbital stealth system is contraindicated when used in coronary arteries, bypass grafts, stents, or where thrombus or dissections are present. Complications associated with OA in CONFIRM registry series were: dissection (11.3\%), spasm $(6.3 \%)$, slow flow (4.4\%), embolism (2.2\%), vessel closure $(1.5 \%)$, thrombus $(1.2 \%)$, and perforation $(0.7 \%) .^{52}$ Other complications reported with OA use are hemolysis and hemolysis-induced pancreatitis. ${ }^{58}$ Clinical significance of hemolysis and OA has been studied in the CLEAR study ${ }^{59}$ which was done in patients with severe peripheral arterial disease. Among 31 study patients, eleven (35.5\%) showed 
laboratory evidence of hemolysis without any clinically significant hemolysis. Lower glomerular filtration rates, calcified plaque, long atherectomy runs, and solid crown selection were independent predictors of hemolysis in this study. ${ }^{59}$

\section{Conclusion}

We have discussed in this article current atherectomy technologies including: SilverHawk directional atherectomy, where the direction can be adjusted, which is useful for eccentric lesions and is used in peripheral arterial disease; RA, where the burr spins concentrically and mostly is used in calcified coronaries; OA, where the crown orbits the wire and is used in both coronary and peripheral arterial disease; excimer laser atherectomy, which ablates the tissue and is used in both coronary and peripheral arterial disease; and Pathway Jetstream PV Atherectomy, which can be used for RA as well as thrombectomy and is used in peripheral arterial disease. As was discussed in the article, these current atherectomy devices have been used efficiently in treatment of coronary and/or peripheral arterial disease, and atherectomy technologies continuously evolve to become even more effective treatment modalities, which hopefully will also be reflected as improved clinical outcomes in the patients.

\section{Disclosure}

The authors report no conflicts of interest in this work.

\section{References}

1. Ramaiah V, Gammon R, Kiesz S, et al. Midterm outcomes from the TALON Registry: treating peripherals with SilverHawk: outcomes collection. J Endovasc Ther. 2006;13:592-602.

2. McKinsey JF, Goldstein L, Khan HU, et al. Novel treatment of patients with lower extremity ischemia: use of percutaneous atherectomy in 579 lesions. Ann Surg. 2008;248(4):519-528.

3. Kandzari DE, Kiesz RS, Allie D, et al. Procedural and clinical outcomes with catheter-based plaque excision in critical limb ischemia. J Endovasc Ther. 2006;13:12-22.

4. Zeller T, Sixt S, Schwarzwälder U, et al. Two-year results after directional atherectomy of infrapopliteal arteries with the SilverHawk device. J Endovasc Ther. 2007;14(2):232-240.

5. Keeling WB, Shames ML, Stone PA, et al. Plaque excision with the Silverhawk catheter: early results in patients with claudication or critical limb ischemia. J Vasc Surg. 2007;45(1):25-31.

6. Sixt S, Rastan A, Beschorner U, et al. Acute and long-term outcome of Silverhawk assisted atherectomy for femoro-popliteal lesions according the TASC II classification: a single-center experience. Vasa. 2010;39(3): 229-236.

7. Shammas NW, Shammas GA, Helou TJ, Voelliger CM, Mrad L, Jerin M. Safety and 1-year revascularization outcome of SilverHawk atherectomy in treating in-stent restenosis of femoropopliteal arteries: a retrospective review from a single center. Cardiovasc Revasc Med. 2012;13(4): 224-227.

8. Garcia LA. Late Breaking Clinical Trials: DEFINITIVE LE 12 month outcomes. Presented at: VIVA 2012; October 9-12; 2012; Las Vegas, NV.

9. Kaid KA, Gopinathapillai R, Qian F, Salvaji M, Wasty N, Cohen M. Analysis of particulate debris after superficial femoral artery atherectomy. J Invasive Cardiol. 2009;21(1):7-10.
10. Akkus NI, Fay M, Varma J. Percutaneous treatment of delayed postatherectomy superficial femoral artery pseudoaneurysm. J Invasive Cardiol. 2012;24(10):E212-E214.

11. Radvany MG, Kiesz RS. Plaque Excision in Management of Lower Extremity Peripheral Arterial Disease with the SilverHawk Atherectomy Catheter. Semin Intervent Radiol. 2008;25(1):11-19.

12. Spencer B, Yeung AC. Rotational Atherectomy: Concepts and Practice. In: Interventional Cardiology. New York: McGraw-Hill; 2007:333-347.

13. Tran T, Brown M, Lasala J. An evidence-based approach to the use of rotational and directional coronary atherectomy in the era of drugeluting stents: when does it make sense? Catheter Cardiovasc Interv. 2008;72(5):650-662.

14. Tomey MI, Kini AS, Sharma SK. Current status of rotational atherectomy. JACC Cardiovasc Interv. 2014;7(4):345-353.

15. Reifart N, Vandormael M, Krajcar M, et al. Randomized comparison of angioplasty of complex coronary lesions at a single center. Excimer Laser, Rotational Atherectomy, and Balloon Angioplasty Comparison (ERBAC) Study. Circulation. 1997;96:91-98.

16. Dill T, Dietz U, Hamm CW, et al. A randomized comparison of balloon angioplasty versus rotational atherectomy in complex coronary lesions (COBRA study). Eur Heart J. 2000;21:1759-1766.

17. Sharma SK, Dangas G, Mehran R, et al. Risk factors for the development of slow flow during rotational coronary atherectomy. Am J Cardiol. 1997;80:219-222.

18. Maclsaac AI, Bass TA, Buchbinder M, et al. High speed rotational atherectomy: outcome in calcified and noncalcified coronary artery lesions. J Am Coll Cardiol. 1995;26:731-736.

19. Kini A, Marmur JD, Duvvuri S, Dangas G, Choudhary S, Sharma SK Rotational atherectomy: Improved procedural outcome with evolution of technique and equipment. Single-center results of first 1,000 patients. Catheter Cardiovasc Interv. 1999;46:305-311.

20. Zeller T, Krankenberg H, Rastan A, et al. Percutaneous rotational and aspiration atherectomy in infrainguinal peripheral arterial occlusive disease: a multicenter pilot study. J Endovasc Ther. 2007;14(3): 357-364.

21. Zeller T, Krankenberg H, Steinkamp H, et al. One-year outcome of percutaneous rotational atherectomy with aspiration in infrainguinal peripheral arterial occlusive disease: the multicenter pathway PVD trial. J Endovasc Ther. 2009;16(6):653-662.

22. Hassan AH, Ako J, Waseda K, et al. Mechanism of lumen gain with a novel rotational aspiration atherectomy system for peripheral arterial disease: examination by intravascular ultrasound. Cardiovasc Revasc Med. 2010;11(3):155-158.

23. Singh T, Koul D, Szpunar S, et al. Tissue removal by ultrasound evaluation (the TRUE study): the Jetstream G2 system postmarket peripheral vascular IVUS study. J Invasive Cardiol. 2011;23(7):269-273.

24. Sixt S, Scheinert D, Rastan A, et al. One-year outcome after percutaneous rotational and aspiration atherectomy in infrainguinal arteries in patient with and without type 2 diabetes mellitus. Ann Vasc Surg. 2011; 25(4):520-529.

25. Shrikhande GV, Khan SZ, Hussain HG, Dayal R, McKinsey JF, Morrissey N. Lesion types and device characteristics that predict distal embolization during percutaneous lower extremity interventions. JVasc Surg. 2011;53(2):347-352.

26. Choy DS. History of lasers in medicine. Thorac Cardiovasc Surg. 1988;36 Suppl 2:114-117.

27. Taylor K, Reiser C. Next generation catheters for excimer laser coronary angioplasty. Lasers Med Sci. 2001;16:133-140.

28. Biamino G. The excimer laser: science fiction fantasy or practical tool? J Endovasc Ther. 2004;11 Supp1 2:II207-II222.

29. Rogers JH, Laird JR. Overview of new technologies for lower extremity revascularization. Circulation. 2007;116(18):2072-2085.

30. Oraevsky AA, Jacques SL, Pettit GH, Saidi IS, Tittel FK, Henry PD. $\mathrm{XeCl}$ laser ablation of atherosclerotic aorta: optical properties and energy pathways. Lasers Surg Med. 1992;12:585-597. 
31. Grundfest WS, Litvack F, Forrester JS, et al. Laser ablation of human atherosclerotic plaque without adjacent tissue injury. $\mathrm{JAm}$ Coll Cardiol. 1985;5:929-933.

32. Geschwind HJ, Boussignac G, Teisseire B, Benhaiem N, Bittoun R, Laurent D. Conditions for effective Nd-YAG laser angioplasty. Br Heart J. 1984;52:484-489.

33. Abela GS, Crea F, Smith W, Pepine CJ, Conti CR. In vitro effects of argon laser radiation on blood: quantitative and morphologic analysis. J Am Coll Cardiol. 1985;5:231-237.

34. Ebersole D, Dahm JB, Das T, et al. Excimer laser revascularization of saphenous vein grafts in acute myocardial infarction. J Invasive Cardiol. 2004;16(4):177-180.

35. Isner JM, Pickering JG, Mosseri M. Laser-induced dissections: pathogenesis and implications for therapy. J Am Coll Cardiol. 1992;19: 1619-1621.

36. van Leeuwen TG, Meertens JH, Velema E, Post MJ, Borst C. Intraluminal vapor bubble induced by excimer laser pulse causes microsecond arterial dilation and invagination leading to extensive wall damage in the rabbit. Circulation. 1993;87(4):1258-1263.

37. Tcheng JE. Saline infusion in excimer laser coronary angioplasty. Semin Interv Cardiol. 1996;1:135-141.

38. CARMEL Excimer Laser Interventional Study Group, Topaz O, Ebersole D, et al. Excimer laser in myocardial infarction: a comparison between STEMI patients with established Q-wave versus patients with non-STEMI (non-Q). Lasers Med Sci. 2008;23(1):1-10.

39. Topaz O, Bernardo NL, Shah R, et al. Effectiveness of excimer laser coronary angioplasty in acute myocardial infarction or in unstable angina pectoris. Am J Cardiol. 2001;87:849-855.

40. Topaz O, Ebersole D, Das T, et al. Excimer laser angioplasty in acute myocardial infarction (the CARMEL multicenter trial). Am J Cardiol. 2004;93:694-701.

41. Giri S, Ito S, Lansky AJ, Mehran R. Clinical and angiographic outcome in the laser angioplasty for restenotic stents (LARS) multicenter registry. Cathet Cardiovasc Intervent. 2001;52(1):24-34.

42. Ben-Dor I, Maluenda G, Pichard AD, et al. The use of excimer laser for complex coronary artery lesions. Cardiovasc Revasc Med. 2011; 12(1):69. e1-e8.

43. Fernandez JP, Hobson AR, McKenzie D, et al. Beyond the balloon: excimer coronary laser atherectomy used alone or in combination with rotational atherectomy in the treatment of chronic total occlusions, non-crossable and non-expansible coronary lesions. EuroIntervention. 2013;9(2):243-250.

44. Giugliano GR, Falcone MW, Mego D, Ebersole D, et al. A prospective multicenter registry of laser therapy for degenerated saphenous vein graft stenosis: the COronary graft Results following Atherectomy with Laser (CORAL) trial. Cardiovasc Revasc Med. 2012;13(2):84-89.

45. Baim DS, Wahr D, George B, et al. Randomized trial of a distal embolic protection device during percutaneous intervention of saphenous vein aorto-coronary bypass grafts. Circulation. 2002;105(11):1285-1290.

46. Laird JF, Zeller T, Gray BH, et al. Limb salvage following laser-assisted angioplasty for critical limb ischemia: results of the LACI multicenter trial. J Endovasc Ther. 2006;13:1-11.

47. Scheinert D, Laird JR Jr, Schröder M, Steinkamp H, Balzer JO, Biamino G. Excimer laser-assisted recanalization of long, chronic superficial femoral artery occlusions. J Endovasc Ther. 2001;8:156-166.
48. Shammas NW, Coiner D, Shammas GA, Christensen L, Dippel EJ, Jerin M. Distal embolic event protection using excimer laser ablation in peripheral vascular interventions: results of the DEEP EMBOLI registry. J Endovasc Ther. 2009;16(2):197-202.

49. Dave RM, Patlola R, Kollmeyer K, et al. Excimer laser recanalization of femoropopliteal lesions and 1-year patency: results of the CELLO registry. J Endovasc Ther. 2009;16(6):665-675

50. Staniloae CS, Korabathina R. Orbital atherectomy: device evolution and clinical data. J Invasive Cardiol. 2014;26(5):215-219.

51. Heuser RR. Treatment of lower extremity vascular disease: the Diamondback 360 degrees Orbital Atherectomy System. Expert Rev Med Devices. 2008;5(3):279-286.

52. Das T, Mustapha J, Indes J, et al. Technique optimization of orbital atherectomy in calcified peripheral lesions of the lower extremities: the CONFIRM series, a prospective multicenter registry. Catheter Cardiovasc Interv. 2014;83(1):115-122.

53. Dattilo R, Himmelstein SI, Cuff RF. The COMPLIANCE $360^{\circ}$ Trial: A Randomized, Prospective, Multicenter, Pilot Study Comparing Acute and Long-Term Results of Orbital Atherectomy to Balloon Angioplasty for Calcified Femoropopliteal Disease. J Invasive Cardiol. 2014;26(8): 355-360.

54. Safian RD, Niazi K, Runyon JP, et al. Orbital atherectomy for infrapopliteal disease: device concept and outcome data for the OASIS trial. Catheter Cardiovasc Interv. 2009;73(3):406-412.

55. Shammas NW, Lam R, Mustapha J, et al. Comparison of orbital atherectomy plus balloon angioplasty vs balloon angioplasty alone in patients with critical limb ischemia: results of the CALCIUM 360 randomized pilot trial. $J$ Endovasc Ther. 2012;19(4):480-488.

56. Parikh K, Chandra P, Choksi N, Khanna P, Chambers J. Safety and feasibility of orbital atherectomy for the treatment of calcified coronary lesions: the ORBIT I trial. Catheter Cardiovasc Interv. 2013;81(7): 1134-1139.

57. Chambers JW, Feldman RL, Himmelstein SI, Bhatheja R, et al. Pivotal trial to evaluate the safety and efficacy of the orbital atherectomy system in treating de novo, severely calcified coronary lesions (ORBIT II). JACC Cardiovasc Interv. 2014;7(5):510-518.

58. Mehta SK, Laster SB. Hemolysis induced pancreatitis after orbital atherectomy in a heavily calcified superficial femoral artery. Catheter Cardiovasc Interv. 2008;72(7):1009-1011.

59. Staniloae CS, Korabathina R, Lane TA, et al. Study to determine the clinical significance of Hemolysis During Orbital AtheRectomy (CLEAR study). J Endovasc Ther. 2011;18(1):57-63.

60. Rotablator ${ }^{\mathrm{TM}}$ Rotational Atherectomy System [webpage on the Internet]. Marlborough: Boston Scientific Corporation; 2014. Available from: http://www.bostonscientific.com/en-US/products/plaque-modification/ rotablator-rotational-atherectomy-system.html. Accessed. August 26, 2014

61. Boston Scientific Acquires the Interventional Division of Bayer [webpage on the Internet]. Warrendale: MEDRAD, INC.; 2014. Available from: http://www.medrad.com/en-us/info/products/Pages/ jetstream-xc-systems.aspx. Accessed. August 26, 2014

62. Optimal Versatility: A Crowning Achievement [webpage on the Internet]. Saint Paul: Cardiovascular Systems Inc.; 2014. Available from: http://www.csi360.com/peripheral-stealth-360-peripheral-orbitalatherectomy-system-crowns. Accessed August 26, 2014
Medical Devices: Evidence and Research

\section{Publish your work in this journal}

Medical Devices: Evidence and Research is an international, peerreviewed, open access journal that focuses on the evidence, technology, research, and expert opinion supporting the use and application of medical devices in the diagnosis, treatment and management of clinical conditions and physiological processes. The identification of novel

\section{Dovepress}

devices and optimal use of existing devices which will lead to improved clinical outcomes and more effective patient management and safety is a key feature. The manuscript management system is completely online and includes a quick and fair peer-review system. Visit http://www. dovepress.com/testimonials.php to read real quotes from authors. 